admissions in a large acute hospital serving a catchment of 650,000

Methods From July 2011 to December 2012, all admissions via the Acute Medical Unit (AMU) were screened using the VitalPAC clinical observation system with a VitalPAC Alcohol Assesment Score (VPAAS) based on the Paddington Alcohol Test. Atrisk patients (VPAAS of 6 or more) were referred to the ASNS and an Alcohol Use Disorders Identification Test (AUDIT) performed. Data analysis was performed on patient demographics, unit consumption, diagnosis, mortality and previous ED attendances and admissions.

Results There were 29,361 admissions of whom 28,098 (96\%) completed VPAAS alcohol screening. Mean AMU population age was 67.4 years, $52.3 \%$ female. Of 1,123 high risk cases, 770 were seen by the ASNS and 636 defined as dependent (AUDIT >20). Compared to the general AMU cohort, the at-risk group had more ED attendances (7.8 vs. 2.9) and hospital admissions (4.8 vs. 3.1) in the previous 3 years and a lower age of death (58.3 vs. 81.5). Dependent women had fewer recurrent attendances and admissions than men but had a higher mortality rate and lower age of death (52.2 vs. 62.4). The maximum AUDIT score of 40 was recorded in $41 \%$ of cases seen by the ASNS and this subgroup had a mean age of death of 52.7 with 6.2 admissions and 10.8 ED attendances previously. The most frequent primary diagnoses in those with a VPAAS of $6+$ were liver disease, mental health disorders and GI bleeding.

Conclusion Our analysis of over 28,000 admissions demonstrates that screening of all medical patients for alcohol misuse is achievable. We successfully identified a cohort of high risk patients with recurrent admissions and ED attendances, high unit consumption and an elevated risk of liver disease and early death. This cohort can be targeted with interventions to reduce the burden of alcohol related harm.

Disclosure of Interest P. Meredith: None Declared, P. Schmidt Conflict with: Unpaid research advisor to The Learning Clinic Ltd that created and licences use of VitalPAC, S. Atkins: None Declared, P. Greengross Conflict with: Part-time Medical Director of The Learning Clinic Ltd that created and licences the use of VitalPAC, G. Westwood: None Declared, R. Aspinall: None Declared.

\section{OC-053 RESULTS OF THE UK MULTI-REGIONAL AUDIT OF BLOOD COMPONENT USE IN CIRRHOSIS}

${ }^{1} \mathrm{~V}$ Jairath*, ${ }^{2} \mathrm{M}$ Desborough, ${ }^{2} \mathrm{~B}$ Hockley, ${ }^{3} \mathrm{M}$ Sekhar, ${ }^{3} \mathrm{~A}$ Burroughs. ${ }^{1}$ Nuffield Department of Medicine, University of Oxford, Oxford, UK; ${ }^{2}$ NHS Blood and Transplant, Oxford, UK; ${ }^{3}$ Royal Free Hospital, London, UK

\subsection{6/gutinl-2014-307263.53}

Introduction Cirrhosis is a complex acquired disorder of coagulation with a recent paradigm shift in understanding to consider cirrhosis as a pro-thrombotic disorder. It is a frequent indication for transfusion of blood components, both for prophylaxis and for treatment of bleeding, although indications and patterns of blood use are poorly characterised.

Methods All NHS trusts with representation on the BSG membership list were invited to take part in a national audit. Data were collected prospectively on conseutive admissions with a confirmed diagnosis of liver cirrhosis over a 4 week period, with follow up to discharge/death/day 28. Specific information was requested on use of blood components, including indication, type of component and laboratory indices prior to transfusion. Standards were defined against guidelines on the use of red blood cells (RBCs), fresh frozen plasma (FFP), platelets and cryoprecipitate.

Results Data on 1313 consecutive patients with cirrhosis (mean age 58 years, 65\% male) were collected from 85 hospitals. The predominant aetiology was alcohol (70\%; 921/1313); $74 \%$ of admissions were for features of decompensation; and $21 \%(275 / 1313)$ cases had a positive septic screen. 30\% (391/ 1313) of all admissions were transfused a blood component; in $61 \%(238 / 391)$ this was for treatment of bleeding and in $39 \%$ (153/391) for prophylaxis. In patients transfused for bleeding (81\%, 192/238 for gastrointestinal bleeding), 92\% (220/238) received RBCs, 32\% (77/238) FFP, 14\% (34/238) platelets and $4 \%(10 / 238)$ cryoprecipitate; in patients with bleeding who received RBCs, the $\mathrm{Hb}$ threshold was $>8 \mathrm{~g} / \mathrm{dL}$ prior to RBC transfusion in 31\% (69/220) cases. For prophylaxis the majority $(61 \%, 94 / 153)$ received transfusion in the absence of a planned procedure. In patients transfused for prophylaxis prior to a procedure (59/153): $19 \% \quad(3 / 16)$ received FFP at an INR $\leq 1.5$ for high risk procedures and $33 \%(6 / 18)$ received FFP at an INR $\leq 2$ for low risk procedures; $36 \%$ (9/25) received platelet transfusion at a platelet count $>50$ prior to a procedure. The most frequent procedures resulting in prophylactic transfusion were paracentesis $(18 / 59)$, surgery (15/59) and endoscopy (10/59). In-hospital venous thromboembolism was documented in 2\% (29/1313) cases. Case fatality during follow up was $10 \%$ overall (128/ $1313)$ with decompensated cirrhosis $(41 \% ; 52 / 128)$ as the most frequent cause of death.

Conclusion Patients with cirrhosis are frequently transfused during hospitalisation. This audit highlights areas where greater scrutiny of blood component use is required, particularly in the group transfused for prophylaxis of bleeding. Further work is needed to improve patterns of blood use in cirrhosis to ensure patients are not exposed to unnecessary transfusion and its attendant harms.

Disclosure of Interest None Declared.

\section{BSG nurses' association/GIN "Education Day" \\ OC-054 THE VALUE OF A WARD LIAISON NURSE TO IMPROVE ENDOSCOPY FOR IN-PATIENTS}

A Gaglia, N Taggart*, L Williams, L Flavell, J Dahill, N Haslam, R Barkatali, S Sarkar. Endoscopy, Royal Liverpool Hospital, Liverpool, UK

\subsection{6/gutjnl-2014-307263.54}

Introduction Endoscopy Inpatients are challenging as they tend to be the sickest patients who require procedures urgently. The numbers fluctuate and there is little provision for capacity in a busy day-case endoscopy unit. At the Royal Liverpool there are $>2000$ in-patient endoscopic procedures per annum and which are served by 9 per week dedicated in-patient lists. However, these lists were poorly utilised at $<50 \%$ of time this was due to cancellations and poor scheduling. Waiting times for inpatients were unacceptable. The aim was to improve the inpatient experience of endoscopy and improve efficiency of the in-patient lists.

Methods In February 2013, an In-patient liaison (B6) was appointed to help coordinate in-patient listing for endoscopy. 